\title{
GSA-ANN and DEA-ANN Methods to Prevent Underground Cable Line Faults
}

\author{
Bahadir Akbal ${ }^{*}$ \\ Department of Electrical and Electronic Engineering, Selçuk University, Konya, Turkey. \\ * Corresponding author. Tel.: +903322232050; email: bakbal@selcuk.edu.tr \\ Manuscript submitted December 13, 2017; accepted March 8, 2018. \\ doi: 10.17706/ijcee.2018.10.2.85-93
}

\begin{abstract}
Cable faults is an important issue for underground cable lines, and the sheath current is a major factor for cable faults. The sheath current occurs on metallic sheath of high voltage underground cable. Thus, metallic sheath of underground cable is grounded to prevent the sheath current faults. If the sheath current which will generate on metallic sheath, the most suitable grounding method can be selected to prevent the sheath current effects. In this study, artificial neural network (ANN) is used to determine the sheath current of underground cable line, and many simulation studies of the sheath current are made to obtain training data of ANN. Simulation studies of sheath current of different underground cable lines are made in PSCAD/EMTDC. Also optimization methods are used to update weights of ANN. Gravitational search algorithm (GSA) and differential evolution algorithm (DEA) are used to optimize weights of ANN. It is seen that training and forecasting errors of hybrid methods are lower than classic ANN.
\end{abstract}

Key words: Artificial neural network, differential evolution algorithm, gravitational search algorithm, underground cable.

\section{Introduction}

High voltage underground cable lines are used to ensure electrical safety in city center and crowded areas because insulation level of underground cable is more than overhead lines. Also recently, overhead lines have been converted to high voltage underground cable lines in city center and crowded areas. Different insulation material are used for insulation of underground cable according to voltage level, and insulation layer is protected by metallic sheath. A high voltage cable layers are shown in Fig. 1 [1]. Metallic sheath protects cable layers against to environmental factors and damages. However, the sheath current is generated on metallic sheath. The sheath current increases cable temperature, so cable insulation life and performance are reduced by the sheath current, also electroshock risk occurs due to high sheath voltage. It is seen that the sheath current must be reduced to provide electrical safety. Thus, metallic sheath is grounded to reduce sheath voltage and current [2]-[5], and bonding methods are used to ground metallic sheath. Single point bonding, solid bonding and cross bonding methods are used as bonding methods in IEEE 575-1988 standard. These bonding methods are shown in Fig. 2, Fig. 3 and Fig. 4.

If cable length is short, single point bonding method can be used, but single point bonding method should be not used for long high voltage underground cable line because the sheath voltage the end of line can be extremely increase. Thus, solid bonding and cross bonding methods are used for long high voltage underground cable line. The sheath current of solid bonding method is less than cross bonding method. Also, sheath current of cross bonding is less than sheath current of solid bonding [6], [7]. Namely, these 
methods can be used according to problem.

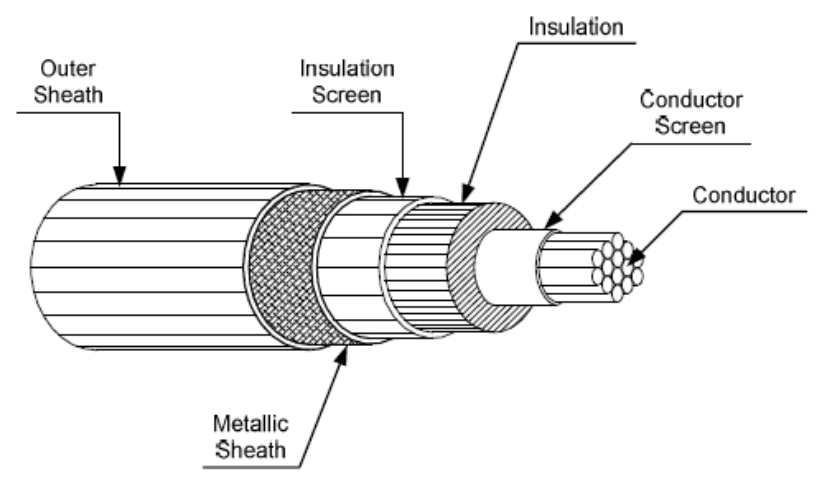

Fig. 1. High voltage underground cable.

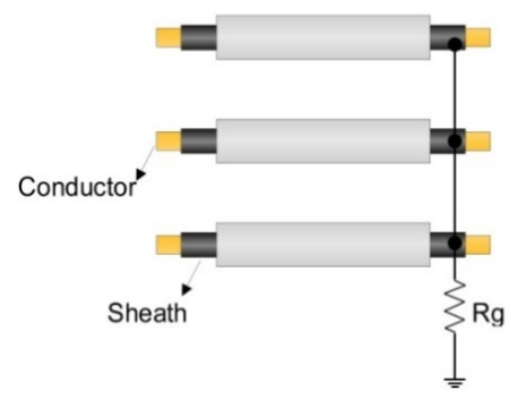

Fig. 2. Single-poind bonding.

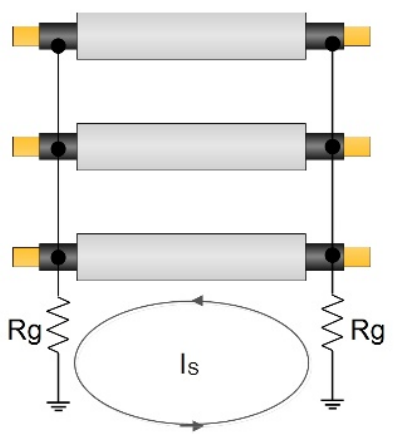

Fig. 3. Solid bonding.

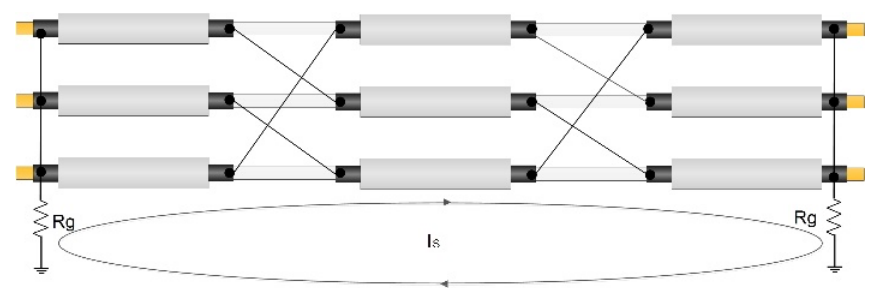

Fig. 4. Cross bonding.

Many simulation studies are made to find the most important factors for formation of the sheath current in PSCAD/EMTDC program. It is seen at the end of simulation studies that cable length $(L)$, distance of between phases $(d)$, unbalanced phase current $\left(I_{U B}\right)$, cable formation and grounding resistance $\left(R_{g}\right)$ are the most important factors for formation of the sheath current in high voltage underground cable line [8]-[11]. 
Forecasting methods can be used to determine the sheath current of high voltage cable line which will be installed as a new line at the project phase, and the most suitable method can be selected to reduce the sheath current effects. Artificial neural network is very useful method for forecasting studies. Hence, in this study artificial neural network based hybrid methods are used to determine the sheath current of high voltage underground cable line. In this study, differential evolution algorithm (DEA) and gravitational search algorithm (GSA) are used for hybrid methods.

\section{Material and Method}

Simulation of high voltage underground cable is made by using electromagnetic transient program [12]. In this study, simulation studies for high voltage underground cable lines are used PSCAD/EMTDC as electromagnetic transient program. In simulation studies of high voltage cable, primarily high voltage cable is modelled, and the modeled cable is shown in Fig. 5.

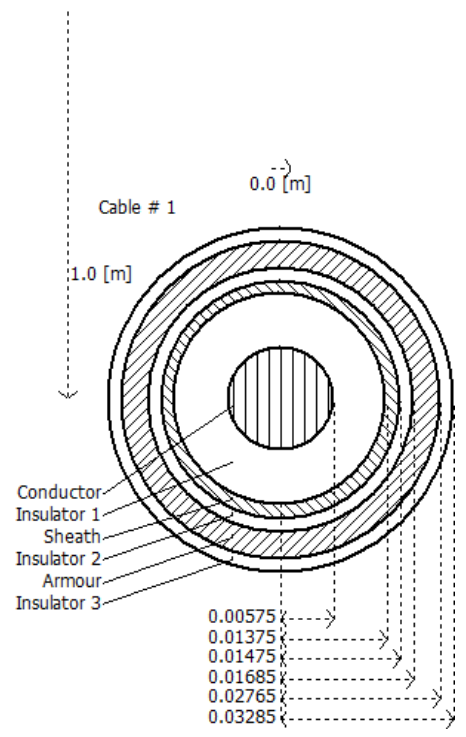

Fig. 5. The modeled high voltage underground cable.

Cable length, distance of between phases, unbalanced phase current and grounding resistance are the most important factors in formation of the sheath current of the high voltage underground cable lines. These factors are used to generate different high voltage cable lines, and simulations of these cable lines are made in PSCAD/EMTDC to obtain sheath currents of these lines. Thus, the real values of sheath current of different lines are used for training process of ANN. Working principle of ANN is based on mathematical model of human learning, and the basic element of ANN is neuron. Also ANN occurs from input layer, hidden layer and output layer [13]-[15]. The basic schema of ANN is shown in Fig. 6.

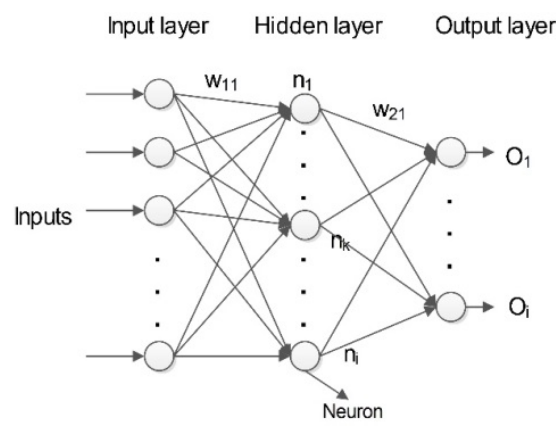

Fig. 6. The basic schema of ANN. 
Neurons work as a transfer function. This transfer function is shown in Equation (1), and a neuron working schema is shown in Fig. 7.

$$
y_{i}=f_{i}\left(\sum_{j=1}^{n} w_{i j} \times x_{j}+b_{i}\right)
$$

where, $x_{j}$ is input, $w_{i j}$ is weight, $b_{i}$ is bias, $f_{i}$ is transfer function, and $y_{i}$ is output of the neuron.

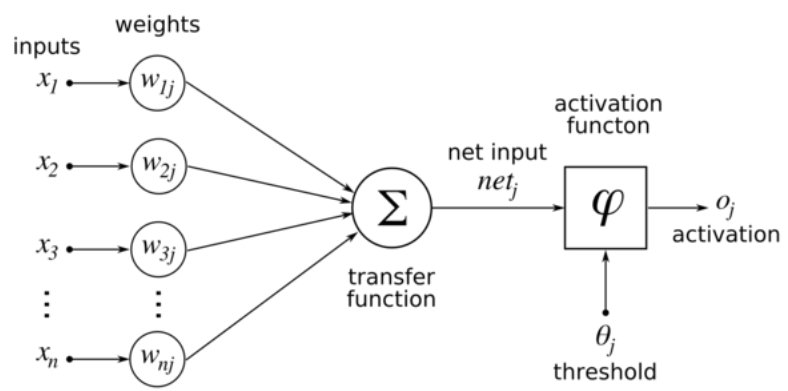

Fig. 7. Neuron working schema.

Activation functions can be sorted as step function, linear function, log-sigmoid function, tan-sigmoid function, softmax function, and gaussian function. Primarily, training process is implemented in ANN, and training error is calculated. Mean square error (MSE) method is used in calculation of training error. MSE is shown in Equation (2).

$$
E(t)=\frac{1}{n} \sum_{i=1}^{n}(p(i)-o(i))^{2}
$$

where, $E(t)$ is estimation error at $t^{\text {th }}$ iteration, $p(i)$ is the desired value for $i^{\text {th }}$ output, $o(i)$ is the actual value for its output.

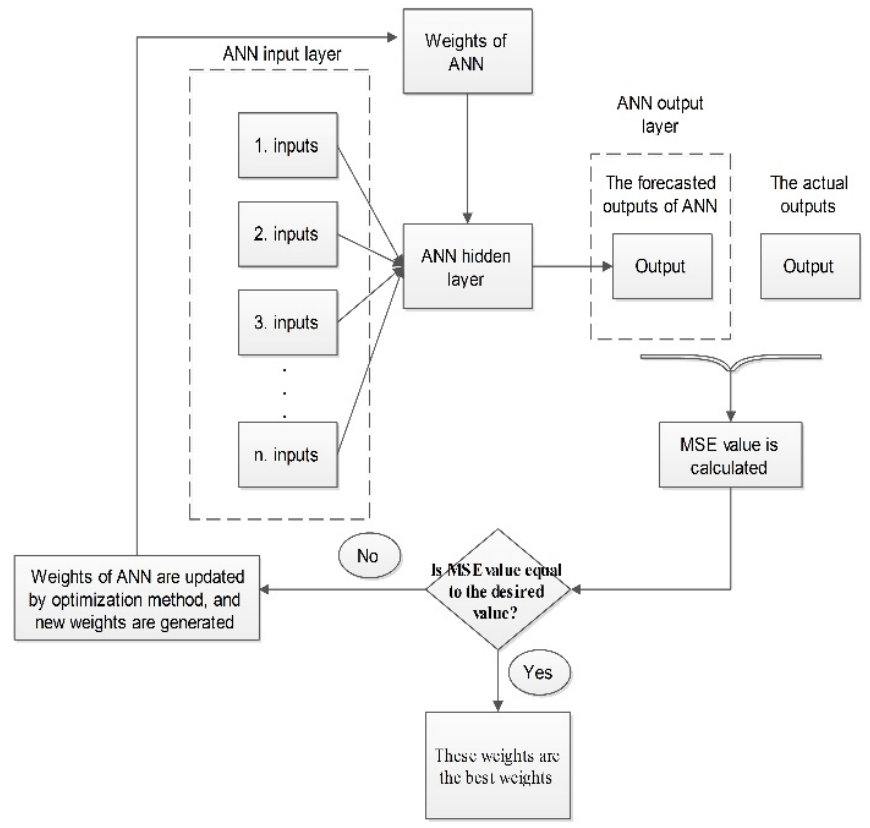

Fig. 8. Hybrid ANN working schema.

Weights of ANN should be updated to reduce training error, so Equation (3) is used to update weights in 
classic ANN.

$$
w_{i}(t+1)=w_{i}(t)+\Delta w_{i}(t)
$$

In hybrid ANN, Equation (3) is not used to update weights, and optimization methods are used instead of Equation (3) to update weights of ANN [16]. Gravitational search algorithm and differential evolution algorithm are used as optimization methods in this study. Hybrid ANN working schema is shown in Fig. 8.

\subsection{Gravitational Search Algorithm (GSA)}

GSA is based on gravitational law which was found by Newton. Each particle which is defined in gravitational law is defined as agent in GSA. According to gravitational law, the other particles are attracted by gravitational force of each particle. Gravitational force is affected by particles masses. The particle which has heavier mass attracts the other particles, and the other particles move towards the particle. In GSA, if agent has heavier mass, fitness value of agent is higher value, and it is near to optimum solution. Each agent has four features. These feature are position $\left(X_{i j}\right)$, inertial mass $\left(M_{i j}\right)$, active gravitational mass $\left(M a_{i}\right)$ and passive gravitational mass $\left(M_{p i}\right) . M a_{i}=M p_{i}=M_{i j}$ is calculated with Equation (4) [17].

$$
\begin{aligned}
m_{i}(t) & =\frac{\operatorname{fit}(t)-\operatorname{worst}(t)}{\operatorname{best}(t)-\operatorname{worst}(t)} \\
M_{i}(t) & =\frac{m_{i}(t)}{\sum_{j=1}^{N} m_{j}(t)} \quad i=1,2, \ldots . N
\end{aligned}
$$

If agent number is $N$ in search space, each agent position can be defined by Equation (6).

$$
X_{i}=\left(x_{i}^{1} \ldots x_{i}^{d} \ldots x_{i}^{n}\right), \quad i=1,2, \ldots . N
$$

$N$ is agent dimension, and agents occur particles. Namely, $N$ is defined as variable of agent. $F$ is gravitational force between $i^{\text {th }}$ agent and $j^{\text {th }}$ agent at the $t$ time. Gravitational force is shown in Equation (7).

$$
x_{i}^{d}(t+1)=x_{i}^{d}(t)+v_{i}^{d}(t+1)
$$

$M_{i}$ is mass of $i^{\text {th }}$ agent, $M_{j}$ is mass of $j^{\text {th }}$ agent, $R_{i j}(t)$ is the Euclidian distance between $i^{\text {th }}$ agent and $j^{\text {th }}$ agent, and it is defined Equation (8). $G(t)$ is gravitational constant and is defined Equation (9).

$$
\begin{aligned}
& R_{i j}(t)=\left\|X_{i}(t), X_{j}(t)\right\|^{2} \\
& G(t)=G_{0} \cdot e^{-\beta\left(t / t_{\max }\right)}
\end{aligned}
$$

$G_{0}$ is an initial value, $\beta$ is constant value, $t$ is the current iteration number, $t^{\max }$ is maximum iteration number, and total force on $i^{\text {th }}$ agent is defined with Equation (10) at $t$ time.

$$
F_{i}^{d}(t)=\sum_{j \in K_{\text {best }}, j \neq i}^{N} \operatorname{rand}_{j} F_{i j}^{d}(t)
$$

rand $_{j}$ is random number between 0 and $1, K_{\text {best }}$ is the first agents which have the best fitness value. Acceleration of agent is calculated with Equation (11). Velocity and position are updated with Equation (12) and Equation (13) according to acceleration of agent at the $t$ time.

$$
a_{i}^{d}(t)=\frac{F_{i}^{d}(t)}{M_{i i}(t)}
$$




$$
\begin{gathered}
v_{i}^{d}(t+1)=\operatorname{rand}_{i} \cdot v_{i}^{d}(t)+a_{i}^{d}(t) \\
x_{i}^{d}(t+1)=x_{i}^{d}(t)+v_{i}^{d}(t+1)
\end{gathered}
$$

$x_{i}^{d}(t) \quad$ is position of $i^{\text {th }} v_{i}^{d}(t)$ agent, is velocity of agent, and $r a n d_{j}$ is random number between 0 and 1 at the $t$ time. Working principle of GSA as follows [14]:

1- Initial population is occurred, and positions of agents are determined as randomly.

2- Fitness values of agents are calculated.

3- Best and worst agents are selected in population, and $\mathrm{G}$ is updated.

4- $M$ and $a$ of agents are calculated, and velocity and position of agents are updated.

After these steps are implemented, fitness values of the updated agents are calculated. If optimum value is found, or the maximum iteration number is reached, program is stopped. Otherwise, the steps which are between 2 and 4 are continuously implemented.

\subsection{Differential Evolution Algorithm (DEA)}

DEA is generally used to solve optimization problems in electrical engineering. Each variable is called as vector in DEA, and there are four type vectors. These vectors are target, base and different two vectors. Namely at least 4 vectors are necessary for DEA.

DEA works as follows [18]:

Step 1: Vector population is randomly generated.

Step 2: Target, base and different two vectors is selected in population. Each vector is respectively selected as target vector in vector population. Base and different two vectors are randomly selected.

Step 3: Difference of between the selected difference two vectors is taken, and base vector is added, so mutant vector is generated.

Step 4: Mutant vector and target vector are used for crossover process. A new trial vector is generated after crossover.

Step 5: Fitness values of target and new trial vectors are calculated, and these values are compared. If fitness value of new trial vector is better than target vector, new trial vector is changed as target vector. Otherwise, target vector is not changed.

Step 6: These processes are implemented each vector, and new vector population is generated.

\section{Experimental Studies}

In this study, DEA and GSA are used for optimization of weights of ANN, so hybrid GSA-ANN and DEA-ANN methods are generated to forecast the sheath current of high voltage underground cable line. In these hybrid ANN methods, population size is 10 , iteration number is 100 . It is seen at the end of high voltage cable simulation studies that unbalanced phase current $\left(I_{U B}\right)$, cable length $(L)$, distance of between phases $(d)$ and grounding resistance $\left(R_{g}\right)$ are the most important factors for generating of the sheath current. Therefore, different high voltage underground cable lines are generated in PSCAD/EMTDC program, and the sheath currents of these lines are measured, so training and test data matrices are generated for hybrid ANN methods. $58 \times 5$ data matrix are used for training of ANN, and forecasting data matrix is shown in Table 1. In Table 1, there are 12 different cable model features for high voltage underground cable lines. The sheath currents of these different cable lines are measured in PSCAD/EMTDC, and the measured sheath current is called as $I_{S A}$. After training process, training errors are obtained these methods, training error of DEA-ANN is 0.2816 , and training error of GSA-ANN is 0.2936 , and training error of classic ANN is 1.8148. Afterward, 
forecasting process is implemented.

Table 1. Different Cable Model Features

\begin{tabular}{cccccc}
\hline \hline Line Model & $I_{U B}(A)$ & $L(m)$ & $d(m)$ & $R_{g}(\Omega)$ & $I_{S A}(A)$ \\
\hline Line 1 & 224.630 & 250 & 0.5 & 5 & 4.00 \\
\hline Line 2 & 224.630 & 250 & 0.5 & 6 & 3.34 \\
\hline Line 3 & 224.630 & 500 & 0.5 & 8 & 4.97 \\
\hline Line 4 & 224.630 & 500 & 0.5 & 9 & 4.42 \\
\hline Line 5 & 224.630 & 500 & 0.5 & 10 & 3.98 \\
\hline Line 6 & 224.630 & 500 & 0.5 & 40 & 1.00 \\
\hline Line 7 & 464.120 & 700 & 0.1 & 40 & 3.32 \\
\hline Line 8 & 464.120 & 500 & 0.5 & 25 & 3.55 \\
\hline Line 9 & 464.120 & 500 & 0.5 & 30 & 2.96 \\
\hline Line 10 & 464.120 & 500 & 0.5 & 35 & 2.54 \\
\hline Line 11 & 464.120 & 500 & 0.5 & 40 & 1.97 \\
\hline Line 12 & 464.120 & 1000 & 0.5 & 40 & 4.36 \\
\hline \hline
\end{tabular}

Table 2. Forecasting Results of the Sheath Current

\begin{tabular}{ccccc}
\hline \multicolumn{3}{c}{$I_{S A}(A)$} & \multicolumn{3}{c}{$I_{S F}(A)$} \\
\hline Line Model & & DEA-ANN & GSA-ANN & ANN \\
\hline Line 1 & 4.00 & 3.12 & 3.14 & 4.54 \\
\hline Line 2 & 3.34 & 3.12 & 3.14 & 4.54 \\
\hline Line 3 & 4.97 & 3.82 & 3.68 & 4.87 \\
\hline Line 4 & 4.42 & 3.81 & 3.68 & 4.63 \\
\hline Line 5 & 3.98 & 3.81 & 3.67 & 4.56 \\
\hline Line 6 & 1.00 & 3.58 & 3.61 & 4.54 \\
\hline Line 7 & 3.32 & 2.90 & 3.69 & 4.54 \\
\hline Line 8 & 3.55 & 2.44 & 3.29 & 4.54 \\
\hline Line 9 & 2.96 & 2.41 & 3.28 & 4.54 \\
\hline Line 10 & 2.54 & 2.37 & 3.27 & 4.54 \\
\hline Line 11 & 1.97 & 2.33 & 3.25 & 4.54 \\
\hline Line 12 & 4.36 & 3.77 & 4.36 & 4.54 \\
\hline \hline
\end{tabular}


Forecasting results of these methods are shown in Table 2, and the forecasted sheath current is called as $I_{S F}$. Forecasting error of DEA-ANN is 0.2223 , and forecasting error of GSA-ANN is 0.2306 , and forecasting error of classic ANN is 1.2233. It is seen that the results of hybrid ANN are better than classic ANN, also the results of DEA-ANN method are better than the results of PSO-ANN method.

\section{Conclusion}

In this study, the sheath current of high voltage underground cable line is examined, and simulations are made in PSCAD/EMTDC. Forecasting of the sheath current is important issue to prevent cable faults of high voltage underground cable lines. Hybrid ANN methods are suggested to determine the sheath current of high voltage underground cable lines which will be installed as a new line. DEA-ANN and GSA-ANN methods are used as hybrid ANN methods. It is seen that the results of hybrid ANN are better than classic ANN. Also the results of DEA-ANN method are better than GSA-ANN method. Hence, the sheath current is forecasted by DEA-ANN method, so the required precautions can be accurately determined.

\section{Acknowledgment}

This study was supported by 17701339 number Selçuk University Coordinatorship of Scientific Research Projects.

\section{References}

[1] Tziouvaras, A. D. (2006). Protection of high-voltage AC cables. Proceedings of the 59th Annual Conference for Protective Relay Engineers (pp. 48-61). TX: College Station.

[2] Benato, R. (2009). Multiconductor analysis of underground power transmission systems: EHV AC cables. Electric Power Systems Research, 79, 27-38.

[3] Ruiz, J. R., Garcia, A., \& Morera, X. A. (2007). Circulating sheath currents in flat formation underground power lines. Proceedings of 2007 International Conference on Renewable Energies and Power Quality (pp. 1-5).

[4] Zhonglei, L., Du, B. X., Wang, L., Yang, C., \& Liu, H. J. (2012). The calculation of circulating current for the single-core cables in smart grid. Proceedings of IEEE Innovative Smart Grid Technologies - Asia (pp. 1-4).

[5] Czapp, S., Dobrzynski, K., Klucznik, J., \& Lubosny, Z. (2014). Calculation of induced sheath voltages in power cables - single circuit system versus double circuit system. Journal of Information, Control and Management Systems, 12, 113-123.

[6] Gouramanis, K. V., Kaloudas, C. G., Papadopoulos, T. A., Papagiannis, G. K., \& Stasinos, E. K. (2011). Sheath voltage calculations in long medium voltage power cables. Proceedings of IEEE 2011 Trondheim Power Tech. (pp. 1-7).

[7] Jung, C. K., Lee, J. B., Kang, J. W., Wang, X. H., \& Song, Y. H. (2005). Sheath current characteristic and its reduction on underground power cable systems. Proceedings of IEEE 2005 Power Engineering Society General Meeting (pp. 2562-2569).

[8] Jung, C. K., Lee, J. B., \& Kang, J. W. (2007). Sheath circulating current analysis of a cross-bonded power cable systems. Journal of Electrical Engineering \& Technology, 2, 320-328.

[9] Gouda, O. E., \& Farag, A. A. (2012). Factors affecting the sheath losses in single-core underground power cables with two-points bonding method. International Journal of Electrical and Computer Engineering, 2, 7-16.

[10] Ruiz, J. R., \& Morera, X. A. (2006). Effects of the circulating sheath currents in the magnetic field generated by an underground power line. Proceedings of International Conference on Renewable Energies and Power Quality (pp. 1-5). 
[11] Moutassem, W., \& Anders, G. J. (2010). Calculation of the eddy current and hysteresis losses in sheathed cables inside a steel pipe. IEEE Transactions on Power Delivery, 25, 2054-2063.

[12] Gudmundsdottir, U. S., Gustavsen, B., Bak, C. L., \& Wiechowski, W. (2011). Field test and simulation of a 400-kV cross-bonded cable system. IEEE Transactions on Power Deliver, 26, 1403-1410.

[13] Charytoniuk, W., \& Chen, M. S. (2000). Very short-term load forecasting using artificial neural networks. IEEE Transactions on Power Systems, 15, 263-268.

[14] Achanta, R. (2012). Long term electric load forecasting using neural networks and support vector machines. International Journal of Computer Science and Technology, 3, 266-269.

[15] Weigerta, T., Tianb, Q., \& Lianb, Q. (2011). State-of-charge prediction of batteries and batterysupercapacitor hybrids using artificial neural networks. Journal of Power Sources, 196, 4061-4066.

[16] Armaghani, D. J., Shoib, R. S., Faizi, K., \& Rashid, A. S. A. (2017, February). Developing a hybrid PSO-ANN model for estimating the ultimate bearing capacity of rock-socketed piles. Neural Comput. \& Applic., 28(2), 391-405.

[17] Rashedi, E., Nezamabadi, H., \& Saryazdi, S. (2009). GSA: A gravitational search algorithm. Elsevier Information Sciences, 179, 2232-2248.

[18] Akbal, B., \& Aydın, M. (2014). DE-Otsu method to eliminate ice load effect. J. Appl. Environ. Biol. Sci., 4, 423-427.

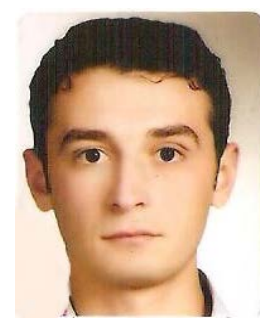

B. Akbal was born in Elbistan, Turkey, in 1983. He received the B.Sc. degree in Electrical-Electronics Engineering Department from Inonu University, Malatya, Turkey in 2006 and M.Sc. degree Electrical-Electronics Engineering Department from Selçuk University, Konya, Turkey in 2011 and then, Ph.D degree Electrical-Electronics Engineering Department from Selçuk University, Konya, Turkey in 2015. He has studied in Electrical-Electronics Engineering Department from Selçuk University as assistant professor Dr. 